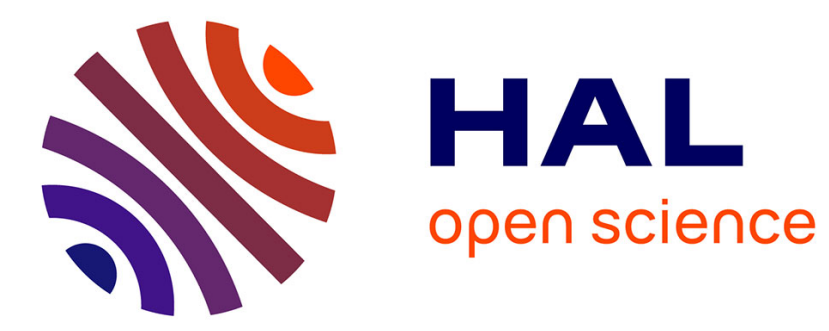

\title{
Les deux récits de création de la femme dans la Genèse
}

Christophe Batsch

\section{To cite this version:}

Christophe Batsch. Les deux récits de création de la femme dans la Genèse. Semitica et Classica, 2012, 5, pp.181-188. 10.1484/J.SEC.1.103055 . hal-01669280

\section{HAL Id: hal-01669280 \\ https: / hal.univ-lille.fr/hal-01669280}

Submitted on 21 Dec 2017

HAL is a multi-disciplinary open access archive for the deposit and dissemination of scientific research documents, whether they are published or not. The documents may come from teaching and research institutions in France or abroad, or from public or private research centers.
L'archive ouverte pluridisciplinaire HAL, est destinée au dépôt et à la diffusion de documents scientifiques de niveau recherche, publiés ou non, émanant des établissements d'enseignement et de recherche français ou étrangers, des laboratoires publics ou privés. 
Les deux récits de création de la femme dans la Genèse

Christophe Batsch

Université de Lille, Laboratoire Cecille

C'est l'inépuisable «question des origines » que les textes fondateurs que nous évoquons ici prétendent aborder. La difficulté n'est pas mince, s'agissant de l'origine de la différenciation sexuée, puisqu'il s'agit de repenser cette question de l'origine, avant et sans la sexualité ; et dans la mesure où n'existe encore ni homme ni femme, il s'agit de penser une origine sans mère ni père, autrement dit de tenter l'impossible.

Le grand texte biblique dont le titre même, dans les différentes langues qui l'ont transmis, Beréchit («en premier»), Genesis ou Genèse, indique qu'il traite de l'affaire des origines témoigne lui aussi, dans sa forme comme dans son contenu, de la difficulté à penser cette origine de la différence sexuelle.

Tout au long de cette étude du texte biblique qui prétend rendre compte de la création des genres sexués, il importera de garder à l'esprit la distinction entre différents moments historiques. Quels que soient les désaccords et les disputes concernant la datation du texte biblique, et en dépit de notre incapacité à établir une chronologie précise, on peut au moins s'accorder sur cette périodisation élémentaire :

$1^{\text {ère }}$ étape. L'élaboration d'un récit mythique de création, ancien, et encore assez enraciné semble-t-il dans le terreau mésopotamien, assyrien ou babylonien.

$2^{\text {ème }}$ étape. La relecture théologique par des cohanim de Jérusalem au début de l'époque du deuxième Temple; la composition finale du texte biblique par juxtaposition du récit primitif et de sa préface théologique.

$3^{\text {ème }}$ étape. La réception du texte biblique et les harmonisations exégétiques des deux récits. Ces interprétations juives et non-juives sont élaborées à la fin de l'époque du deuxième Temple et à l'époque talmudique.

Pour en venir au texte de la Genèse, et d'abord à sa forme, la critique textuelle a fermement établi qu'à un premier récit de la création de l'être humain et de sa différenciation entre hommes et femmes, fut ajouté ensuite un deuxième récit, généralement présenté comme « sacerdotal » (source P), c'est-à-dire daté de l'époque du rétablissement du culte à Jérusalem, 
après l'exil babylonien. ${ }^{1}$ Le récit le plus ancien (Genèse 2, 4-7) est donc désigné comme présacerdotal ou, selon une appellation plus ancienne, comme « yahwiste » (source J) et apparaît par endroit encore proche de ses sources mésopotamiennes. ${ }^{2}$

Le récit le plus récent a été ajouté postérieurement, comme une sorte de préface conceptuelle et théologique, de sorte qu'il apparaît paradoxalement le premier dans l'ordre narratif : ce sont le premier chapitre de la Genèse et les trois premiers versets du deuxième chapitre (Gn 1 et $2,1-3)$.

À cette redondance de la forme, il faut associer une multiplication des personnages sur le fond : en effet, selon la tradition juive, ce ne sont pas moins de trois figures de «première femme » que la Genèse met en scène dans ces récits. Cette multiplication des personnages de femme originelle témoigne assez, à mon sens, de la difficulté de penser la question des origines hors-une-femme, c'est-à-dire de penser une origine sans mère. On ne retrouve pas pareilles hésitations dans les autres épisodes de la création rapportés dans la Genèse. La mécanique anthropologique de la création par séparation et opposition des éléments deux-àdeux y fonctionne en effet plus facilement : création-opposition du ciel et de la terre, de la lumière et des ténèbres, du jour et de la nuit, du sec et de l'humide, de la végétation et des animaux, de la nature et de l'humanité, toutes ces opérations fonctionnent impeccablement. Comment la machine en vient-elle à se gripper lorsqu'il s'agit de créer la différence entre un homme et une femme ?

Il doit être bien clair que lorsque nous parlons ici de «machine» ou de «mécanique » c'est d'opérations textuelles, mythiques et narratives dont il est exclusivement question. C'est dans le texte lui-même que nous repérons donc blocages et difficultés au sujet de la création de la différence sexuée.

Considérons nos deux récits dans l'ordre où ils furent produits.

Dans le plus ancien (Genèse 2, 7-8, complété par Genèse 2, 15. 18-22) :

1. L'homme est fabriqué à partir de «la poussière de la terre » 'afar min-ha damah et du « souffle de vie » nishmat hayyîm, Gn 2, 7 ; l'homme est seul et seulement masculin.

2. Il est placé dans le gan-eden «pour le travailler et pour le préserver » $l^{e}$ 'abdah $w l^{e}$ shamrah, Gn 2, 15.

3. Dieu crée les animaux « car il n'est pas bon que l'homme soit seul », Gn 2, 18-20.

\footnotetext{
${ }^{1}$ Voir, inter al., C. UEHLINGER, «Genèse 1-11 », T. RÖMER, J.-D. MACCHI et C. NIHAN (éd.), Introduction à l'Ancien Testament, Genève, 2004, p. 114-133.

${ }^{2}$ Plus précisément $\mathrm{C}$. Uehlinger isole un noyau primitif proche de ces sources (en Gn 2, 5 à 2, 7aa) : «Le récit "J primitif" se situe assez près de l'épopée d'Astrahasis, de Gilgamesh XII et d'une version sumérienne du déluge », ibid., p. 118.
} 
4. La femme est fabriquée ensuite, dans un deuxième temps, et à partir du corps de l'homme (Gn 2, 21-22).

Dans le plus récent, issu de l'école sacerdotale (Gn 1, 21-28 complété par Gn 5, 1-2) :

1. les animaux sont créés avant l'homme $\left(5^{\text {ème }}\right.$ et $6^{\text {ème }}$ jour $)$ «selon leur espèce » $l^{e}$ mînah, (LXX : kata genos), Gn 1, 21. 24-25.

2. L'homme est créé par Dieu (Elohim) «à l'image de Dieu », Gn 1, 27.

3. Dès l'origine coexistent masculin et féminin (androgynie primitive), Gn 1, 27 et 5, 2.

4. Toute puissance est donnée à cet être sur le monde ici-bas, Gn 1,28 : « soumettez-la (i.e. la terre) et dominez » kibshuha $w r^{e}$ dî etc.

Il peut être légitime de replacer ces deux récits dans l'ordre final selon lequel ils ont été reçus, repris et commentés. Concernant précisément la différenciation des sexes, on est alors confronté à ces deux séquences :

1. L'androgynie fondamentale de l'humanité dans le récit sacerdotal : à l'origine coexistent dans le même être le masculin et le féminin, « selon l'image de Dieu » (Gn 1, 27 et 5, 2).

2. Puis, de façon toute différente dans le récit ancien, la fabrication subsidiaire de la femme à partir du corps du premier homme, pour lui servir d'aide et de compagne (Gn 2, 18-22).

Ces différences, incompatibilités, voire contradictions, entre les deux récits, même et peutêtre surtout lorsqu'ils étaient reçus comme un seul et même récit, ont très tôt été perçues et les traditions juives se sont très tôt efforcées d'harmoniser toute l'histoire.

À la fin de l'époque du deuxième Temple et au début de l'ère commune, on peut ainsi observer deux tentatives parallèles d'harmonisation au sein du judaïsme.

La première est celle de Philon d'Alexandrie. Le dossier sur ce sujet est assez bien connu et je ne m’y étendrai pas. Philon interprète la succession des deux récits à sa façon allégorique et platonisante. On peut résumer, de façon un peu succincte, le développement de sa pensée en trois points :

$1^{\mathrm{er}}$ point : Le récit de Genèse 1 rend compte de la création d'une sorte d'archétype idéal et spirituel de l'être humain.

$2^{\text {ème }}$ point : Cette première création concerne le genre humain dans son ensemble, donc un être humain au sens le plus général et le plus indifférencié : «Ayant donné à l'espèce (to genos) le nom d'homme (anthrôpon), Moïse en distingua les genres (ta eidê) en disant que (Dieu) l'avait créé mâle et femelle (arren te kai thêly), bien que les éléments sexués (tôn en 
merei) n'eussent pas encore été formés. ${ }^{3}$ Philon suppose donc un être humain générique en quelque sorte antérieur à la différenciation des sexes. ${ }^{4}$

$3^{\text {ème }}$ point : Les deux récits de création correspondent à deux états de la créature : d'abord l'archétype idéal de l'humanité. Puis le deuxième récit montre la création du premier homme réel et charnel, bientôt différencié en premier couple sexué, homme et femme. Dans le vocabulaire de Philon c'est le premier homme « sensible », aisthêtos.

Je ne fais ici que rappeler les grandes lignes d'une réflexion de Philon, qu'il développe et élabore à plusieurs reprises. 5

Cette harmonisation platonicienne du récit de la création des genres semble avoir poursuivi son chemin au sein de la gnose. On la retrouve en effet presque à l'identique sous la plume évidemment critique d'Irénée de Lyon quand il décrits les croyances des gnostiques rattachés à la figure de Marcos : «À moins qu'ils ne disent que l'homme "choïque" a été modelé le sixième jour et l'homme charnel le huitième jour : car ils distinguent ces deux choses. Certains prétendent aussi distinguer l'homme à la fois mâle et femelle fait à l'image et à la ressemblance de Dieu - ce serait l'homme "pneumatique" - et l'homme modelé au moyen de la terre. ${ }^{6}$

On y retrouve deux points essentiels : d'une part l'harmonisation par la différenciation diachronique des deux récits de création, replacés respectivement au $6^{\text {ème }}$ jour et au $8^{\text {ème }}$ jour du monde. D'autre part la distinction introduite entre l'homme « charnel » (carnalis) ou «modelé de terre» (lat.: ex terra plasmatu: gr.: ek tês gês plathenta); et l'homme spiritualisé, «choïque » (choicum, choikos) ou «pneumatique » (spiritalis, pneumatikos). L'emploi du terme choicum (choikos) reflète la complexité des constructions gnostiques et peut-être aussi la difficulté d'Irénée à en rendre compte ; en effet, il désigne un création à partir d'un limon liquide (choos). L'interprétation générale de ce mot a toujours été d'y voir un quasi synonyme de «hylique ». Tel que l'ont défini A. Rousseau et L. Doutreau, l'homme «choïque » est un être fabriqué «non de la terre sèche mais de la substance invisible, de la fluidité et de l'inconsistance de la matière (...) dans le système gnostique exposé par Irénée,

\footnotetext{
${ }^{3}$ De opificio mundi 76. Ma traduction.

${ }^{4}$ Cette opération d'escamotage de la question sexuelle a suscité, entre autres, l'ironie de Daniel Boyarin : In other words, the designation of this creature as male-and-female means really neither male nor female, $\mathrm{D}$. Boyarin, Carnal Israel. Reading Sex in Talmudic Culture, Berkeley, 1993, p. 38. («En d'autres termes la qualification de cette créature comme mâle et femelle signifie en réalité : ni mâle, ni femelle. »)

${ }^{5}$ On se reportera pour l'essentiel aux passages suivants : De opificio mundi, 24-25; 69-88; 134 ; 151-152. Legum allegoriae I 32-42; II 4 ;13. De Cherubim, 59-60. Quis rerum divinarum heres sit, 139-140; 164. Quaestiones in Genesim I 25.

${ }^{6}$ Adversus Haereses I, 18, 2. Traduction A. Rousseau et L. DoutreAu, Irénée de Lyon. Contre les Hérésies, livre I, tome II, Paris, (Sources chrétiennes 264), 1979, in loc.
} 
choïque est pratiquement synonyme de hylique : l'homme hylique, proche de Dieu mais sans lui être consubstantiel $»^{7}$ En d'autres termes : même idéalisé et «à l'image de Dieu », l'homme pneumatique est aussi composé de matière. Mais il est probable que la matière identifiée par le terme choos soit plus «noble» que la poussière dont est fait l'homme charnel, en particulier en raison des connotations liquides associée à ce terme. Il est d'autant plus intéressant de constater que, dans cette tradition gnostique conservée par Irénée, cet homme choïque, c'est-à-dire à la fois spirituel, fait à l'image de Dieu et matériellement créé, se révèle androgyne : masculino-feminus ou arsenothelyn.

Malheureusement la documentation nous manque pour décider si cette androgynie est définie, comme chez Philon par son absence: l'homme choïque serait alors moins androgyne qu'asexué, ne possédant que sous une forme seulement encore virtuelle les potentialités sexuelles des deux genres. Ou si cette androgynie se définit de manière positive, par la possession des attributs des deux sexes.

Un passage bien connu de l'Évangile copte de Philippe peut donner à penser que ce système gnostique prenait en compte une androgynie sexuellement complète. Il donne en tout cas une représentation positive de cette androgynie primitive, puisqu'elle est associée à l'immortalité : «Quand Ève était [en] A[d]am, la mort n'existait pas. Quand elle fut séparée de lui la mort survint.-À nouveau, du moment qu'il est entré et qu'elle l'a reçu en elle, la mort doit cesser. ${ }^{8}$ L'élément nouveau réside ici en ce que ce lien entre androgynie et immortalité réapparaîtra à la fin des temps. Il a donc existé, pour autant qu'on puisse en juger sur d'aussi faibles bases textuelles, un espoir (ou une aspiration) gnostique de retrouver le corps pneumatique, immortel et androgyne de la première création : c'est surtout cette dimension physique et corporelle de l'androgynie qui distingue les élaborations gnostiques de la réflexion de Philon.

Il reste que cette représentation androgyne du premier être humain, conçu comme parfait et semblable à l'image de Dieu, aurait pu (ou aurait dû) conduire à une représentation égalitaire des deux sexes. On sait qu'il n'en a rien été. Dans l'exégèse de Philon, le drash (allégorique) vient ici corriger ce que le pshat (sens littéral) aurait de trop évidemment égalitaire. Philon admet volontiers que le sens littéral de Gn 2, 21-22 est clair (littera manifesta est) : « homme et femme (vir et mulier), en tant que parties de la nature, sont rigoureusement égaux dans la

\footnotetext{
${ }^{7}$ A. Rousseau et L. Doutreau, Irénée de Lyon (supra n. 6)

${ }^{8}$ Nag Hammadi II,3 $\$ 71$ (116, 22-26). Traduction L. PAINCHAUd, J.-P. MAHE et P.-H. PoIRIER (éd.), Écrits gnostiques. La bibliothèque de Nag Hammadi, Paris, (Pléiade), 2007, p. 335-376.
} 
formation du genre, qui est appelé "Homme" (Homo). » ${ }^{9}$ Mais, il y a un « mais », ad mentem vero etc.: «mais au sens allégorique », l'homme représente l'intellect et la femme la puissance sensible ; et celui-la vaut le double de celle-ci. ${ }^{10}$

La tradition chrétienne, au moins à partir d'Augustin, ira plus loin encore en rejetant toute représentation bisexuée de l'être humain primitif et en privilégiant le récit le plus ancien de la création de la femme, à partir d'un des côtés d'Adam, bientôt réduit à une simple côte. ${ }^{11}$

On trouve dans la tradition rabbinique un effort similaire d'harmoniser les deux récits de la Genèse, mais par des moyens et avec des résultats bien différents.

Première différence essentielle : la tradition juive a toujours admis l'androgynie primitive de l'être humain, reflet de la plénitude divine dont il est l'image. Dans la réflexion des Sages, on ne peut évidemment pas faire intervenir des notions de critique littéraire contemporaine ; on est dans le moment de la réception d'un texte révélé et faisant autorité, tel qu'il se présente. Le récit de la différenciation sexuelle s'inscrit dès lors entre deux affirmations de l'androgynie primitive, « à l'image de Dieu »: Gn 1, 27 et Gn 5, 2. Ces deux versets sont très proches dans leur formulation.

Gn 1, 27 : «Alors Elohim créa l'Adam à son image, à l'image d'Elohim il le créa ; mâle et femelle il les créa ».

Gn 5, 2: «Mâle et femelle il les créa ; puis il les bénit et il cria leur nom “Adam” en ce jour qu'il les créa ».

Je ne reviendrai pas ici sur les nombreuses attestations d'une variante textuelle qui établit encore plus clairement l'unicité androgyne de l'être primitif puisqu'on y lit: «Mâle et femelle il le créa. »C'est une chose désormais connue. ${ }^{12}$

\footnotetext{
${ }^{9}$ Quaestionnes et solutiones in Genesim I 25 sur Gn 2, 21-22.

${ }^{10}$ Voir aussi De Cherubim 59-60 : «The mind (ekeinos) was docked of all its powers of sense-perception (aisthetika), thus truly powerless. It was but half the perfect soul (psyché) (...) God then, wishing to provide [the mind] with perception of material as well as immaterial things, sought to complete the soul by weaving into the part first made the other section, which he called by the general name of 'woman' and the proper name of "Eve", thus symbolizing sense (aisthesis). "

${ }^{11}$ Voir en particulier Augustin, De Trinitate livre XII, VI, 8, où l'auteur prétend imposer une ponctuation correcte au verset Genèse 1,27. Serge Cazelais a montré que ce rappel à l'ordre visait à exclure l'interprétation d'un Adam primitif «prétendu androgyne » (quos androgynos uocant) ou monstrueusement « hermaphrodite » (quasi monstruosum aliquid sicuti sunt quos hermaphroditos uocant), S. CAZELAIS, « La masculoféminité d'Adam : quelques témoins textuels et exégèses chrétiennes anciennes de Gen. 1,27 », Revue Biblique 114/2, 2007, p. 174-188. La condamnation par Augustin de la thèse d'un Adam primitif androgyne apparaît également dans le De Genesis ad litteram livre III, XXII, 34

${ }^{12}$ Voir mon article C. BATSCH, «Les sexes du jardinier. Quelques spéculations des Anciens sur l'androgynie d'Adam », dans D. Barbu et al. éd., Mondes clos. Cultures et jardins, Lausanne : Éditeurs Infolio (Suppléments Asdiwal 1), 2013, p. 223-239 \& 365-366 ; et S. CAZELAIS, « La masculoféminité d'Adam... » (supra n. 11).
} 
Le point essentiel et retenu par les Sages du Talmud est celui-ci : l'être humain primitif est androgyne, à l'image de Dieu, et son nom unique d'Adam indique qu'il fut modelé d'une seule et même glèbe. Cette théorie de l'androgynie primitive d'un être humain unique nommé Adam est développée en plusieurs endroits de la littérature rabbinique classique.

Elle s'élabore dans les débats talmudiques sur la nature d'un Adam à double face, par exemple en bErouvin 18a: «R. Jérémie ben Eléazar dit: Adam avait un double visage de faces (dio partsouf panim). " Cette double nature d'Adam (dont les Sages trouvent la justification exégétique dans le Psaume 139, 5) est à nouveau évoquée dans un débat exégétique sur les orthographes différentes du verbe yitser («modeler») selon qu'il s'applique à la création de l'homme (avec deux yods, Gn 2, 7) ou à la création des animaux (avec un seul yod, Gn 2, 19). Rappelons que ce geste du «modelage » désigne l'acte même de la création. Un premier Sage suggère que les deux yods sont destinés à « montrer que le Saint, béni soit-il, a créé deux tendances (en l'homme), l'une au bien, l'autre au mal. » Cette opinion est aussitôt rejetée par la majorité. La juste exégèse suggère plutôt que ces deux yods expriment la double nature de l'Adam primitif, « selon l'explication donnée par R. Jérémie ben Eléazar ; car R. Jérémie ben Eléazar dit : Le Saint, béni soit-il, a créé deux visages (diprotsopin, du grec diprosôpos) dans le premier Adam, comme il est dit Tu m'a formé devant et derrière (Ps 139, 5). »13

Environ la même époque, ${ }^{14}$ le midrach Berechit Rabba 8, 1 développant le récit de la création d'Adam et sa différenciation ultérieure en deux sexes, fixe la théorie de l'androgynie primitive: «Puis Elohim dit: Faisons l'homme à notre image, selon notre ressemblance (Gn 1, 26) (...) R. Yeremyah ben Léazar dit : quand le Saint béni soit-il fit le premier Adam il le créa androgyne ('andrôgînos, décalque du mot grec), comme il est dit : mâle et femelle il les créa $(\mathrm{Gn} 5,2)$. R. Shmouel bar Nahman dit : quand le Saint béni soit-il fit le premier Adam, il le créa double-face (diprosopon ${ }^{15}$ ) puis il le sépara et fit un dos pour ceci et un dos pour cela. On objectera: Il est pourtant écrit: YHWH... prit une de ses côtes ('ahat missal 'otâw, Gn 2, 21). R. Shmouel bar Nahman répond : une de ses côtes signifie l'un de ses deux côtés, selon les mots et pour la côte du Tabernacle (Ex 26, 20) que le targoum traduit en araméen et pour le côté du Tabernacle. »

\footnotetext{
${ }^{13}$ bBerakhot 61a. Selon une interprétation de Rab Yehudah (b Ketubot 8a) : «Au commencement il était dans l'intention (de Dieu) de créer deux (êtres) mais finalement un seul fut créé. »

${ }^{14}$ Sur la dation du Berechit Rabba, cette note de Nicholas De Lange : «Le plus ancien, en toute probabilité, de ces midrachim dits aggadiques, le Midrach sur la Genèse (Beréchit Rabba) fut rédigé (de l'avis de presque tous les spécialistes) vers la fin $\mathrm{du} \mathrm{V}^{\mathrm{e}}$ siècle, en Palestine byzantine. » N. DE LANGE, « Midrach et Byzance. Une traduction française du "Midrach Rabba". Notes critiques », Revue de l'histoire des religions 206/2, 1989, p. 171-181.

${ }^{15}$ Un terme analogue au di-protsopin de bBerakhot 61a et bErubin 18a, l'un et l'autre calqués sur le grec.
} 
Grâce à cet argument linguistique qui substitue « le côté » à «la côte », l'harmonisation des deux récits est obtenue : l'être humain primitif est d'abord androgyne (suivant le récit sacerdotal); il est ensuite séparé en ses deux moitiés sexuées (suivant le récit mythique). Toute la tradition juive ultérieure se développe à partir de là.

Il importe de mesurer tout ce que cette tradition recèle de potentiel d'ambivalence, s'agissant des relations entre les sexes.

D'un côté, la femme et l'homme sont issus de la même création initiale, du premier modelage d'une identique argile; il n'y a donc pas d'antériorité de l'un par rapport à l'autre, pas non plus de supériorité généalogique ou générique du masculin comme dans les traditions ayant privilégié le récit de « la côte d'Adam ».

D'un autre côté, la division de l'être primitif en ses deux genres est indiscutablement un affaiblissement. Dans la mesure où cet affaiblissement de l'être humain primitif découle de la division en deux genres, il se trouve donc associé à la création de la femme. Objectivement il est tout aussi lié à la création du genre masculin, mais cet aspect a été gommé par le fait qu'une continuité formelle fut établie entre le nom Adam de l'être primitif et le nom Adam de l'homme masculin séparé de sa part féminine.

Plusieurs passages de la littérature rabbinique classique expriment cette théorie sous-jacente que la division de l'être humain primitif en un couple distinct et sexué a correspondu à un affaiblissement de sa nature. Cet affaiblissement s'est révélé nécessaire et fut voulu par la divinité car l'être qu'il avait créé (à son image) s'est révélé dangereusement trop parfait. Pour le midrach (Berechit Rabba 8, 10) même les êtres célestes ont pu s'y tromper : "Quand le Saint, béni soit-il, eut créé Adam le premier homme, les anges du service se méprirent sur son compte et voulurent clamer devant lui "Saint". » ${ }^{16}$ Plus tard, le Pirqé deRabbi Éliézer 11 étend cette dangereuse confusion aux créatures terrestres : «Lorsque l'homme se dressa sur ses jambes, son aspect était à la ressemblance d'Elohim. Les créatures en le voyant s'effrayèrent car elle le prirent pour leur Créateur et toutes vinrent se prosterner devant lui. » ${ }^{17}$ Qu'est-ce qui a rendu possible cette confusion a priori impensable entre le Créateur et sa créature ? C'est que l'Adam, à l'image de Dieu, est un. Cette unité essentielle se manifeste dans ses deux attributs que sont d'une part l'immortalité, d'autre part son corollaire, l'absence

\footnotetext{
${ }^{16}$ Traduction B. MARUANI et A. COHEN-ARAZI, Lagrasse, 1987, comme les autres citations de ce midrach.

${ }^{17}$ Traduction M.-A. OUAKNINE et É. SMILEVITCH, Lagrasse, 1983, comme les autres citations de ce midrach.
} 
de mécanisme de reproduction. ${ }^{18}$ Logiquement c'est donc par la suppression de ces deux attributs que sera opérée la correction. Une aggada du Talmud de Babylone l'illustre, tout en rapprochant cette réduction à l'état humain de l'Adam primitif de l'ambition insensée des rois proche-orientaux et de leur prétention à être considérés comme des dieux : «Rab Judah déclare au nom de Rab : Le Saint, béni soit-il, dit à Hiram roi de Tyr : [Lors de la création] je t'ai vu [te prendre pour un dieu ${ }^{19}$ ], aussi j'ai créé à l'homme plusieurs orifices. D'autres disent: je t'ai vu et j'ai condamné Adam à la mort» (bBaba Bathra 75a-b). Les deux modifications évoquées ici ne se contredisent pas mais se complètent. L'être humain, parce que désormais mortel, doit par conséquent se nourrir et se reproduire. Y pourvoient les nombreux orifices dont il est alors muni. La création de ces orifices a souvent été comprise seulement comme un rappel des fonctions excrémentielles ; celles-ci font partie du processus, sans doute. Mais tous les orifices et toutes les fonctions de ces orifices sont également concernés, qui servent aussi à l'absorption de nourriture et à la reproduction.

Le midrach ne laisse aucun doute que le moment où ces limites furent imposées au statut de l'être humain primitif correspond au moment de la séparation des genres sexués. Ainsi, selon le midrach rabba de la Genèse : «Que fit le Saint, béni soit-il (après avoir observé la méprise des êtres célestes) ? Il l'accabla [i.e., Adam] d'une torpeur et tous surent que ce n'était qu'un homme » (Berechit Rabba 8, 10). Certes, le midrach cite ensuite un verset d'Isaïe concernant la faiblesse constitutive de l'être humain. ${ }^{20}$ Mais il est impossible d'ignorer ici la référence explicite à Genèse 2, 21-22 c'est-à-dire au récit de la division d'Adam en deux être sexués : «Alors YHWH Elohim fit tomber un profond sommeil sur l'Adam qui s'endormit. » Le Pirqé deRabbi Éliézer va plus loin qui suggère que la réflexion prêtée dans la Bible à la divinité, «il n'est pas bon que l'homme soit seul » $(\mathrm{Gn} 2,18)$, découle directement du constat que l'unicité de l'Adam primitif risquait de susciter la confusion entre la créature et son créateur. ${ }^{21}$ On sait que dans le récit biblique, c'est cette réflexion qui conduit à la séparation des sexes.

La phrase de Genèse 2, 18 se poursuit par une conclusion qui a donné lieu à d'innombrables exégèses : «Il n'est pas bon que l’Adam soit seul, juge donc la divinité. Je lui fabriquerai un

\footnotetext{
${ }^{18}$ Voir inter al. le Pirqé deRabbi Éliézer 12 : «Le Saint, béni soit-il, se dit : je suis unique en mon monde et l'homme est unique dans le sien. Il n'y a devant moi ni fructification ni accroissement et l'homme connait la même situation ; par la suite, les créatures pourraient se dire, désignant l'homme : puisque celui-là n'a devant lui ni fructification ni accroissement, c'est lui qui nous a créées. »

19 Voir Ézéchiel 28, 2-10. En particulier Ézéchiel 28, 2 : «Fils d'Adam dis au prince de Tyr: ainsi parle Monseigneur YHWH : parce que ton cœur s'est élevé et que tu as dis "Je suis un dieu, je siège au siège des dieux, au cœur des océans" ; mais tu es un Adam et non un dieu » etc.

${ }^{20}$ Isaïe 2, 22 : «Abandonnez l'homme qui ne tient qu'à un souffle dans les narines, car pour quoi compte-il ?»

${ }^{21}$ Voir Pirqé deRabbi Éliézer 12.
} 
‘ézer $k^{e} n e g d o »$. Cet 'ézer $k^{e} n e g d o$ constitue une sorte d'oxymoron; je le traduirai par « une aide à l'encontre de soi. »

Le mot dont on discute ici est le second $: k^{e} n e g d o$. La racine NGD renvoie à la visibilité, au fait d'être-là, en face de, face à etc. D'où le sens obvie de neged, d'abord comme substantif, puis comme adverbe : celui qui est en face, que l'on voit ; ainsi que celui qui est opposé, qui est contre, donc hostile. Mais il existe aussi un sens dérivé : celui qui est proche, donc facile à vivre. D'où l'existence d'une difficulté pointée par les Sages : «Rabbi Éléazar a relevé une contradiction (raméy) », déclare le Talmud de Babylone (bYebamot 63a).

En définitive, cette «aide» attendue, qui prendra la forme de la femme, va-t-elle être avec ou contre l'homme ? Et qu'est-ce que c'est que cette «aide » ('ézer) qui peut se révéler tout à tour pour et contre l'Adam ? Certaines traditions semblent même envisager que l'être humain primitif aurait pu se trouver un complément existentiel dans le règne animal. Cette réflexion s'inspire du récit de Genèse 2, 18-20: Dieu songe à pourvoir l'homme d'une compagnie ; puis il crée les animaux et les fait défiler devant Adam pour qu'il les nomme ; et le verset 20 se termine ainsi : «(...) mais pour l'Adam il ne trouva pas de 'ézer $k^{e} n e g d o$. »Certains ont pu comprendre que si l'on n'avait pas découvert cette compagnie dans le monde animal c'est qu'on l'y avait cherché ; ou du moins que la reproduction sexuée des animaux avait servi de modèle. Ainsi le midrach conserve-t-il cette observation de Rabbi Aha : «Le Saint, béni soit il, fit à nouveau défiler les animaux devant Adam, ${ }^{22}$ mais cette fois par couple. - Chacun a son partenaire, s'écria Adam, sauf moi : mais pour l'homme il ne trouva pas de 'ézer $k^{e} n e g d o$ (Gn 2, 20). » Il est vrai que cette remarque est accompagnée d'un commentaire à la tonalité nettement misogyne.

Mais l'interprétation dominante de cette « aide à l'encontre de soi » est celle que l'on retrouve jusque chez Rachi : «Si l'homme se comporte avec mérite, elle lui sera une aide. S'il ne le fait pas, elle sera contre lui et le combattra. ${ }^{23}$ Dans cette représentation, qui est devenue la norme, le deuxième être qui doit naître de la séparation de l'Adam primitif en ses deux composantes sexuées sera donc fondamentalement ambivalent, susceptible de soutenir comme de combattre.

Il me semble que cette ambivalence, naturellement attribuée à la part féminine issue de la séparation, répondait à un premier effort théorique de rendre compte de «l'inquiétante

\footnotetext{
${ }^{22}$ Après qu'il les a nommés, selon Gn 2, 19-20.

${ }^{23}$ Rachi, commentaire in loc. Gn 2, 18. Pour les sources rabbiniques, voir bYebamot 63a ; Berechit Rabba 17, 3 ; et Pirqé deRabbi Éliézer 12.
} 
étrangeté » féminine. L'ambivalence fondamentale et constitutive du personnage de la femme attendue, exprimait une forme d'effroi devant la menace égalitaire implicite dans le récit de la création. Car sur quoi fonder la prétention d'un sexe à dominer l'autre, si l'un et l'autre sont issus du même modelage initial ? Une fois de plus le mécanisme narratif et théorique consiste à supposer une continuité de l'être entre l'Adam primitif et sa forme masculine issue de la séparation des sexes. L'autre genre sexué issu de cette séparation, la femme, est ainsi étrangement dissocié de cette continuité et doit se voir attribuer un nouveau nom. Mais l'étrangeté de cette manipulation rhétorique et conceptuelle ne pouvait manquer de ressurgir ; elle le fait sous la forme de l'indécision radicale caractérisant les rapports entre les deux sexes.

Cette première élaboration théorique se poursuit avec le développement de l'exégèse rabbinique sur un autre point du récit de création. Cette exégèse conduit les Sages à introduire dans le récit une première Ève, rejetée par Adam et aussitôt éliminée.

Ce qui suscite les commentaires rabbiniques, c'est l'exclamation de l'être masculin issu de la séparation (et toujours nommé «l'Adam ») lorsque, à son réveil, il découvre sa partenaire féminine : «Et l'Adam dit : Cette fois-ci, c'est l'os de mes os » etc. (Gn 2, 23). L'exclamation a attiré l'attention des Sages : si Adam parle de «cette fois-ci » ( $z$ 'ot happa 'am), c'est donc qu'il y a eu une première fois, une première tentative de séparer les deux sexes qui n'avait pas abouti. Le midrach s'empare de cette hypothétique première fois et en fournit une explication : «(Dieu) commença par créer la femme (sous les yeux de l'homme) mais celuici, la voyant pleine de sécrétions et de sang, s'en écarta. Aussi le Saint, béni soit-il, se reprenant, la créa une seconde fois. » (Berechit Rabba 17, 7). Récit repris presqu'à l'identique un peu plus loin: «Rabbi Yehouda Bar Rabbi dit: Le Saint béni soit-il avait créé une première femme, mais l'homme, la voyant pleine de sang et de sécrétions, s'en était écarté. Aussi le Saint béni soit-il s'y est repris et lui en a créé une seconde » (Berechit Rabba 18, 4). ${ }^{24}$ En d'autres termes, lors de la fabrication de la première Ève l'homme, resté éveillé, a pu assister à tout un processus (apparemment assez analogue à la description de la résurrection des ossements en Ézéchiel 37, 5-10) au cours duquel les organes intérieurs sont d'abord assemblés avant d'être finalement recouverts de la peau.

On se situe ici dans une représentation qui se place au-delà de l'effroi égalitaire, tenu à distance, puisque le récit ne se présente pas comme celui de la séparation de l'être humain primitif en ses deux parties mais comme celui d'une création secondaire de la femme. Ici la

\footnotetext{
${ }^{24}$ Cf. aussi Bereshit Rabba 22, 7.
} 
crainte de l'étrangeté féminine s'est déplacée du terrain de la psychologie à celui de la biologie. À travers le syntagme du «sang et des sécrétions », c'est explicitement la crainte née de l'incompréhension des mystères biologiques de la féminité (en simplifiant : cycles, fécondité, jouissance) qui est évoquée dans ce texte. Il n'est pas inutile de souligner combien, dans un système de représentation symbolique fondé sur la séparation du pur et de l'impur, ces références au sang et aux écoulements faisaient basculer la biologie féminine du côté de l'impureté.

Par la suite la tradition juive, rabbinique et populaire, en viendra à élaborer le personnage de Lilith, par lequel un terme est mis à toute ambivalence puisque Lilith, cette première femme antérieures au deux Èves, permet d'incarner à elle seule toute la représentation négative des femmes : dominatrice, et volontairement stérile, elle prend figure de démone, succube et tueuse d'enfants. Construite à partir d'éléments linguistiques et démonologiques d'inspiration babylonienne (le lilu, la lilitu et l'ardat lili) ${ }^{25}$, tirés de la Bible hébraïque (Isaïe 34,14$)^{26}$ et du Talmud babylonien, elle est tardivement incorporée au récit de la création de l'humanité. Un midrach intitulé Sefer Ben Sira ou Alphabet de Ben Sira, rédigé vers le $\mathrm{X}^{\mathrm{e}}$ siècle, est le premier texte connu qui mette vraiment en scène Lilith comme une proto-Ève. Ce nom qu'on lui prête est celui d'une classe de démons femelles mentionnées dans le Talmud. ${ }^{27}$

Avec l'invention de Lilith, toute revendication d'égalité entre les sexes est repoussée. Celle-ci est en effet associée au premier récit de la création, celui de Genèse 1, 27 : «mâle et femelle il les créa. »C'est précisément parce qu'elle fut créée en même temps et à l'égale de l'homme que Lilith se rebelle contre la domination que celui-ci prétend lui imposer, puis contre les ordres de la divinité, et finit par incarner toutes les menaces d'une féminité hostile et dangereuse, au premier rang desquelles figure la stérilité. On connait le récit qu'en donne le Sefer Ben Sira : «Elle dit : je ne me couche pas au-dessous, et il dit : je ne me couche pas audessous mais dessus car tu es faite pour le dessous et moi pour le dessus. Elle lui dit : nous sommes tous deux égaux puisque nous sommes tous deux de la terre; et ils ne s'écoutaient pas l'un l'autre. Quand Lilith vit cela elle dit le Nom ineffable et s'envola dans l'air. » Autour de cette figure de femme révoltée se développe ensuite toute un appareil légendaire et

\footnotetext{
${ }^{25}$ Il est généralement admis aujourd'hui que le vocable hébraïque lilit est emprunté aux langues assyrobablyloniennes. Le mot dérive du sumérien lil qui signifie «vent» et que l'on trouve dans le nom des Lils, démons akkadiens.

${ }^{26}$ Isaïe 34,14 : « Les bêtes du désert s'assembleront avec les hyènes, le bouc appellera son compagnon ; juste là Lillith reposera et trouvera son repos. » Cf. les traductions anciennes : dans la Septante à la place de la Lilith hébreu, on trouve la figure de «l'onocentaure » (onokentauros) moitié âne et moitié homme ; tandis qu'en latin Saint Jérôme traduit Lilith par lamia, figure d'une démone grecque dévoreuse d'enfants.

${ }^{27}$ Voir aussi Otsar ha-Midrachim, I, 47.
} 
démonologique qui fait de Lilith la responsable de la mortalité infantile, qu'on ne peut combattre qu'au moyen de diverses amulettes invoquant les noms des trois anges protecteurs. Cette pratique était encore largement attestée dans les communautés juives du XIX ${ }^{\mathrm{e}} \mathrm{s}$. Par contraste avec cette figure démoniaque dévoreuse d'enfants, Ève n'est plus que ézer (aide) et fécondité ; elle est pleinement devenue la «la-vivante» (Hawa) et «la mère de tous les vivants » ('em kol hä̈, Gn 3, 20).

Ce sont donc bien trois figures de «première femme» qui surgissent de la tradition juive : dans l'ordre du récit, apparaît d'abord la Lilith égalitaire réfugiée dans l'univers des démons ; puis la proto-Ève biologique créée sous les yeux d'Adam et aussitôt détruite ${ }^{28}$; enfin Ève elle-même, soumise et féconde, créée pendant le sommeil d'Adam.

On ne doit cependant pas tenir pour définitive cette opposition entre une Lilith égalitaire donc stérile et démoniaque et une Ève soumise, féconde et maternelle. Cette représentation populaire, et porteuse de tous les préjugés misogynes, n'est pas celle du judaïsme le plus exigeant. Il apparaît au contraire que les élaborations des savants cabbalistes ont, en leur temps, rétabli la promesse d'une égalité réelle entre les sexes, fondée sur leur propre lecture du récit de la création. Sans nous y étendre plus longuement, concluons donc ici par ce beau texte du Zohar: «Mâle et femelle il les créa (Gn 5, 2). Rabbi Siméon dit: Des secrets suprêmes ont été divulgués par ces deux versets. Le fait qu'il créa Adam mâle et femelle vise à faire connaître la gloire suprême, secret de la confiance, car du sein de ce secret l'homme a été créé. (...) De ce verset nous apprenons que toute figure qui ne comporte pas le masculin et le féminin n'est pas une figure suprême parfaite. Nous l'avons compris grâce au sens intime de la Michna. Viens et vois : partout où le masculin et le féminin ne se trouvent pas ensemble, le Saint, béni soit-il, ne place pas sa résidence en un pareil lieu. Les bénédictions ne résident que dans un lieu où il y a masculin et féminin, ce qu'exprime le verset suivant : Il les bénit et il appela leur nom Adam, au jour de leur création $(\mathrm{Gn} 5,2)$. Mais il n'est pas écrit : Il le bénit et il appela son nom Adam, car même Adam ne se nomme qu'en tant que masculin et féminin réuni. ${ }^{29}$

\footnotetext{
${ }^{28}$ Bereshit Rabba 22, 7 : « Yehouda Bar Rabbi dit: [Caïn et Abel] se querellaient pour [la possession] de la première Ève. Rabbi Ayvou a objecté : Cette première Ėve était déjà retournée à la poussière ».

${ }^{29}$ Zohar Berechit III, 55b. Traduction C. MOPSIK et B. MARUANI, Lagrasse, 1981.
} 
Bibliographie

M. BITTON, «Lilith ou la première Ève : un mythe juif tardif », Archives de Sciences sociales des Religions, 71, 1990, p. 113-136

J. M. BlaIR, De-Demonizing the Old Testament. An Investigation of Azazel, Lilith, Deber, Qeteb and Reshef in the Hebrew Bible, Tubingue, 2009, (Forschungen zum Alten Testament 2.Reihe 37)

D. Boyarin, Carnal Israel. Reading Sex in Talmudic Culture, Berkeley, 1993

J. BRIL, Lilith ou la mère obscure, Paris, 1981

C. CARmichael, Sex and Religion in the Bible, New Haven, 2010

S. CAZELAIS, «La masculoféminité d'Adam: quelques témoins textuels et exégèses chrétiennes anciennes de Gen. 1, 27 », Revue Biblique 114/2, 2007, p. 174-188

J. J. Collins, «Before the Fall: The Earliest Interpretation of Adam and Eve », H. NAJMAN et J. Newman (éd.), The Idea of Biblical Interpretation. Essays in Honor of James Kugel, Leyde, 2004 (Supplement to the Journal for the Study of Judaism 83), p. 293-308

S. FREEMANN, «Lilith au sein du mysticisme juif », http://www.kabbale.eu/lilith-au-sein-dumysticisme-juif/, 2009

M. Goff, «Adam, the Angels and Eternal Life: Genesis 1-3 in the Wisdom of Solomon and 4QInstruction », G. XERAVITS et J. ZSENGELLER (éd.), Studies in the Book of Wisdom, Leyde, 2010, p. 1-21

M. HARL (éd.), La Bible d'Alexandrie 1. La Genèse, Paris, 1986

C. HerRenSCHMIDT, «Anthropogonies graphiques», De Kêmi à Birit Nâri. Revue Internationale de l'Orient Ancien 1, 2003, p. 117-131

S. HuRWITZ, Lilith - die erste Eva, eine Studie über dunkle Aspekte des Weiblichen, Zürich, 1980

I. LEVI, « Lilit et Lilin » dans Revue des études juives 68, 1914

J. R. LeVInson, Portraits of Adam in Early Judaism. From Sirach to 2 Baruch, Sheffield, 1988

W. A. MeEKs, «The Image of the Androgyne. Some Uses of a Symbol in Earliest Christianity », dans A. Hilton et G. Snyder éd., W. Meeks: In Search of the Early Christians, New Haven, 2002, p. 3-54 (1 ${ }^{\text {ère }}$ 1974, History of Religion 13, p. 165-208)

M. E. MONDRIAAN, «Lilith and Eve: Wives of Adam », Old Testament Essay 18/3, 2005, p. $752-762$

D. Morel-Vergniol, «Adam, Ève... et Lilith ? », Cahier biblique 39, 2000, p. 39-51

S. REINACH, « La naissance d'Ève », Revue de l'histoire des religions 77, 1918, p. 185-206

V. Rousseau, «Lilith : une androgynie oubliée», Archives de Sciences sociales des Religions 123, 2003, p. 61-75

C. Uehlinger, «Genèse 1-11», dans T. Römer, J.-D. MACChi et C. NihAn (éd.), Introduction à l'Ancien Testament, Genève, 2004, p. 114-133 